\title{
Microwave Synthesis and Photocatalytic Activity of Cadmium Indium Sulfide Nanocomposite
}

\author{
Kun Tu, Xiaobin Wu, Congsen Wang, Xue-Hong Liao*, Shuibin Yang* \\ Hubei Key Laboratory for Processing and Application of Catalytic Materials, the College of Chemical Engineering, Huanggang \\ Normal University, Huanggang, China \\ Email: *liaoxuehong@sohu.com, ^2416975012@qq.com
}

How to cite this paper: Tu, K., Wu X.B., Wang C.S., Liao, X.-H. and Yang, S.B. (2017) Microwave Synthesis and Photocatalytic Activity of Cadmium Indium Sulfide Nanocomposite. Modern Research in $\mathrm{Ca}$ talysis, 6, 73-79.

https://doi.org/10.4236/mrc.2017.62006

Received: September 12, 2016

Accepted: April 11, 2017

Published: April 14, 2017

Copyright $\odot 2017$ by authors and Scientific Research Publishing Inc. This work is licensed under the Creative Commons Attribution International License (CC BY 4.0). http://creativecommons.org/licenses/by/4.0/

\begin{abstract}
Nanocomposite of $\mathrm{CdIn}_{2} \mathrm{~S}_{4}$ was synthesized by direct feeding microwave synthesis method, using indium nitrate, cadmium nitrate and thioacetamide as raw material, cetyltrimethyl ammonium bromide(CTAB) as surfactant. The crystal structure, morphology and the optical property of as-prepared sample were characterized by X-ray diffraction (XRD) and scanning electron microscope (SEM) and Fluorescence spectra. The results showed that the as-prepared nanocomposite is hexagonal $\mathrm{CdIn}_{2} \mathrm{~S}_{4}$. The SEM showed that the shape of these nanoparticles is irregular and looks like flake/sphere with some aggregation. The size of most of the aggregate is 100 to $300 \mathrm{~nm}$. The photocatalytic activity of the as-prepared samples was studied by using degradation of methylene bule under visible light. The results show that the photocatalytic activity of $\mathrm{CaIn}_{2} \mathrm{~S}_{4}$ photocatalyst was very well. When the catalyst was $1.0 \mathrm{~g} / \mathrm{L}, \mathrm{C}\left(\mathrm{H}_{2} \mathrm{O}_{2}\right)$ was $3 \mathrm{~mL} / \mathrm{L}$, after $120 \mathrm{~min}$ of the irradiation, the degradation rate of $\mathrm{CdIn}_{2} \mathrm{~S}_{4}$ for methylene blue of $20 \mathrm{mg} / \mathrm{L}$ reached $86.06 \%$.
\end{abstract}

\section{Keywords}

Nanocomposite Cadmium Indium Sulfide, Microwave Synthesis,

Photocatalysis, Visible Light, Degradation, Methylene Bule

\section{Introduction}

The future of modern society will depend on how we solve the urgent environmental and energy issues that we face today. Ever since the decomposition of water on a $\mathrm{TiO}_{2}$ electrode under UV light irradiation was first reported in 1972 [1], photocatalytic processes based on semiconductors have offered the most promising solutions. They can both be used for water splitting to produce hy- 
drogen and oxygen and for the degradation of toxic pollutants in wastewater treatment. $\mathrm{TiO}_{2}$ is by far the most widely used and investigated photocatalyst due to its high efficiency and photostability. In addition, it is non-toxic and commercially available at a low price. However, $\mathrm{TiO}_{2}$ has a major drawback that has not been overcome till date. As its band gap is rather wide $(3.2 \mathrm{eV}), \mathrm{TiO}_{2}$ needs to be activated through UV irradiation that represents only $4 \%$ of the energy of the sunlight arriving on the earth's surface. Therefore, the development of visible-light-driven photocatalysts has become one of the most challenging and urgent topics over the past decades. Recent progress in the field has demonstrated that the development of functionalized ternary and higher stability is an efficient strategy to overcome the intrinsic limitations of $\mathrm{MIn}_{2} \mathrm{~S}_{4}$.

$\mathrm{CdIn}_{2} \mathrm{~S}_{4}$ ternary sulfide because of its narrow band gap (2.1 - $\left.2.6 \mathrm{ev}\right)$, there is a strong absorption in the visible region, it posses unique photoelectric properties and catalytic performance [2]-[8]. Ternary metal sulfide $\mathrm{CdIn}_{2} \mathrm{~S}_{4}$ belongs to the $\mathrm{AB}_{2} \mathrm{X}_{4}$ family compound semiconductor compound. The ternary semiconductor due to the narrow band gap and large specific surface area and pore structure, the sufficient contact between the catalyst and the reactant is ensured. It is conducive to the rapid migration and separation of the surface charge of the reactant can effectively inhibit the photogenerated electrons and holes of the composite, improves the photocatalytic efficiency. Binary sulfide is easy to produce light corrosion, the service life is limited, but the ternary sulfide has good stability. At present, several techniques were used to synthesize $\operatorname{CdIn}_{2} \mathrm{~S}_{4}$, such as solventhermal (hydrothermal), high temperature decomposition, electrochemical method and so on.

In this study, we report on a direct feeding microwave synthesis method to synthesize $\mathrm{CdIn}_{2} \mathrm{~S}_{4}$ nanophotocatalyst. The visible-light-driven photocatalytic activity of the $\mathrm{CdIn}_{2} \mathrm{~S}_{4}$ nanophotocatalyst was evaluated with respect to methylene blue photodegradation.

\section{The Experiment}

\subsection{Synthesis}

All chemicals were analytical grade and used without further purification. $\mathrm{CdIn}_{2} \mathrm{~S}_{4}$ materials were prepared by a direct feeding microwave synthesis method. In a typical procedure, $2.0193 \mathrm{~g}$ of $\mathrm{Cd}\left(\mathrm{NO}_{3}\right)_{2} \cdot 4 \mathrm{H}_{2} \mathrm{O}, 5.0000 \mathrm{~g}$ of $\mathrm{In}\left(\mathrm{NO}_{3}\right)_{3} \cdot 4.5 \mathrm{H}_{2} \mathrm{O}$ were added by stoichiometric ratio, double excess of thioacetamide and $1 \mathrm{~g}$ of $\mathrm{CTAB}$ were dissolved in $100 \mathrm{ml}$ of deionized water. Then the mixed solution was placed in a microwave refluxing system to react for $20 \mathrm{~min}$ with a power microwave radiation of $40 \%$ and cool down naturally to the room temperature. Then the precipitate was centrifuged, washed with the deionized water for several times and dried at $60^{\circ} \mathrm{C}$ in the vacuum for 4 hours. The final product was collected for the characterization.

\subsection{Characterization}

The crystal structure of photocatalyst was measured by XRD on a Shimadzu 
$\mathrm{XRD}-6100 \mathrm{X}$-ray diffractometer $(\mathrm{Cu} \mathrm{Ka}$ radiation, $\lambda=0.15418 \mathrm{~nm}$ ). The morphology and size of products were determined by SEM. The SEM images were recorded on a Quanta 200 FEG field emission scanning electron microscope. The optical property was obtained by Cary Eclipse fluorescence spectrometer (USA Varian Company).

\subsection{Photocatalytic Experiments}

The photocatalytic activities of the synthesized powders were evaluated by the degradation $100 \mathrm{ml}$ of $20 \mathrm{mg} / \mathrm{l}$ of methylene bule in $0.1 \mathrm{~g}$ of $\mathrm{CdIn}_{2} \mathrm{~S}_{4}$ photocatayst mixture aqueous suspension under visible-light irradiation. First, the mixed solution was placed in the dark, placed 30 minutes to reach the adsorption equilibrium. Then, in the process of photodegradation, every $10 \mathrm{~min}$, the absorbance of the solution was determined by the UV-visible absorption spectrometer. The photocatalysis reaction lasted until the discoloration of the solution was fulfilled or stopped. The formula of the degradation ratio is as follow [9]

$$
\mathrm{D}_{\mathrm{t}} \%=\left(\mathrm{A}_{0}-\mathrm{A}_{\mathrm{t}}\right) / \mathrm{A}_{0} \times 100 \% \text {. }
$$

\section{Results and Discussion}

Figure 1 shows the XRD pattern of as-prepared sample. The XRD pattern (peak 20: $14.44^{\circ}, 23.62^{\circ}, 27.64^{\circ}, 28.82^{\circ}, 33.46^{\circ}, 41.12^{\circ}, 43.76^{\circ}, 47.82^{\circ}, 55.88^{\circ}, 66.48^{\circ}$ ) showed that the product is the cubic spinel phase of $\mathrm{CdIn}_{2} \mathrm{~S}_{4}$ (JCPDS File No. 27-0060). The diffraction peak is strong and sharp, which indicates that the sample has a high degree of crystallinity.

Figure 2 shows the SEM graph of as-prepared sample. The shape of these nanoparticles is irregular and looks like flake/sphere with some aggregation. The size of most of the aggregate is 100 to $300 \mathrm{~nm}$. There are some small particles scattered around.

Figure 3 is a fluorescence spectra of as-prepared sample, the excitation wavelength is $387 \mathrm{~nm}$. It can be seen that have be provided with a certain photoluminescence properties in $400-600 \mathrm{~nm}$. The luminescence max intensity



Figure 1. X-ray diffraction pattern of as-prepared sample. 


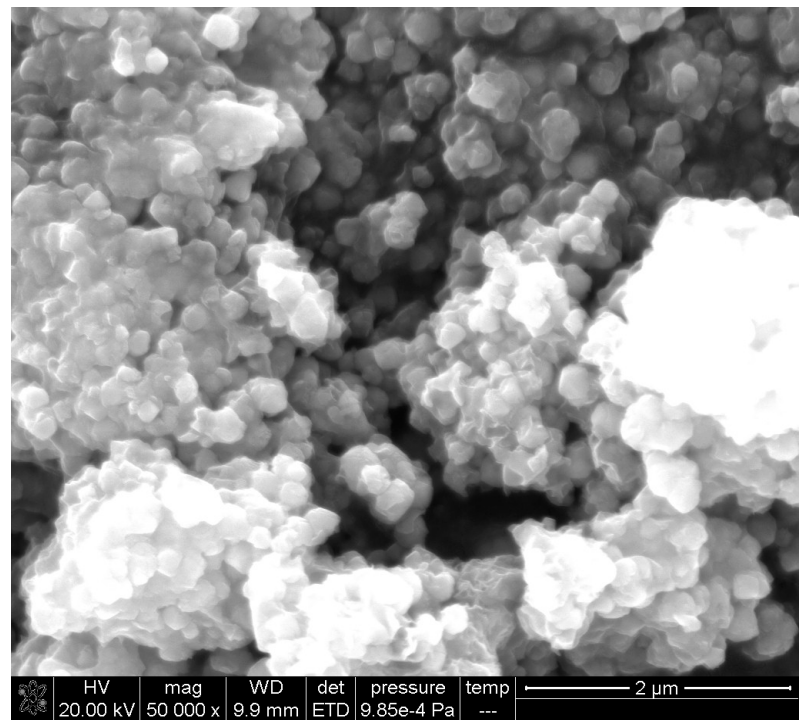

Figure 2. TEM of as-prepared sample.

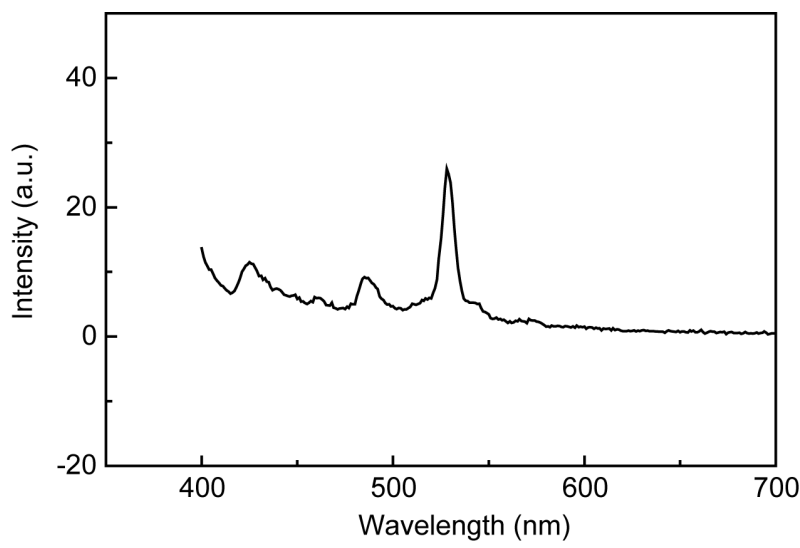

Figure 3. Fluorescence spectra of as-prepared sample.

observed at $528 \mathrm{~nm}$. There are relatively weak luminescence peak in $400-500$ $\mathrm{nm}$.

Figure 4 shows the typical visible absorption spectra of the photo-degradation for the methylene bule. It can be seen that the absorbance of the solution decreases rapidly along with the photocatalytic degradation reaction. After $120 \mathrm{~min}$ of the irradiation, the solution in the range of the visible light has almost no absorption, indicating that the methylene bule has been degraded thoroughly. Reaction of the first 10 minutes, the degradation rate is $50 \%$. Reaction to $40 \mathrm{mi}-$ nutes, the degradation rate is $75 \%$. Reaction to 70 minutes, the degradation rate is $80 \%$. Reaction to 120 minutes, the degradation rate is $86.06 \%$. When the methylene bule solution is directly put under the illumination without the catalyst, the results show that the solution does not fade by continuous illumination for 2 $\mathrm{h}$, and the detection by the UV-visible absorption spectrometer finds that the absorbance does not change. $\mathrm{CdIn}_{2} \mathrm{~S}_{4}$ nanophotocatalyst can catalyze the degradation of methylene blue solution in a short time, which shows that the photocatalytic activity of the prepared catalyst is good. 
The absorbance of methylene blue solution was gradually decreased with the increase of light time, but the maximum absorption wavelength of methylene blue solution was blue shifted. Because of the conjugated structure of methylene blue, in the process of degradation, it may slowly be destroyed, resulting in the maximum absorption of methylene blue solution blue shift.

Figure 5 is the effect of the amount of the nanophotocatalyst on photocatalytic activity. As it can be seen from the figure, the most suitable amount of catalyst is $1.0 \mathrm{~g} / \mathrm{L}$. Too much or too little catalyst is unfavorable for the degradation of methyl orange solution. The amount of catalyst is too little, the use of visible light is not sufficient, the catalytic degradation rate is very slow, long time is required for the degradation. Excessive amount of catalyst leads to precipitation of the solution at the bottom results in waste and may also produce a masking effect of the incident light in the course of the reaction. The light is not sufficiently transmitted to the solution, affecting the full utilization of energy and resulting in photocatalytic efficiency on the decrease. Therefore, the right amount of catalyst is able to fully improve the photocatalytic efficiency.

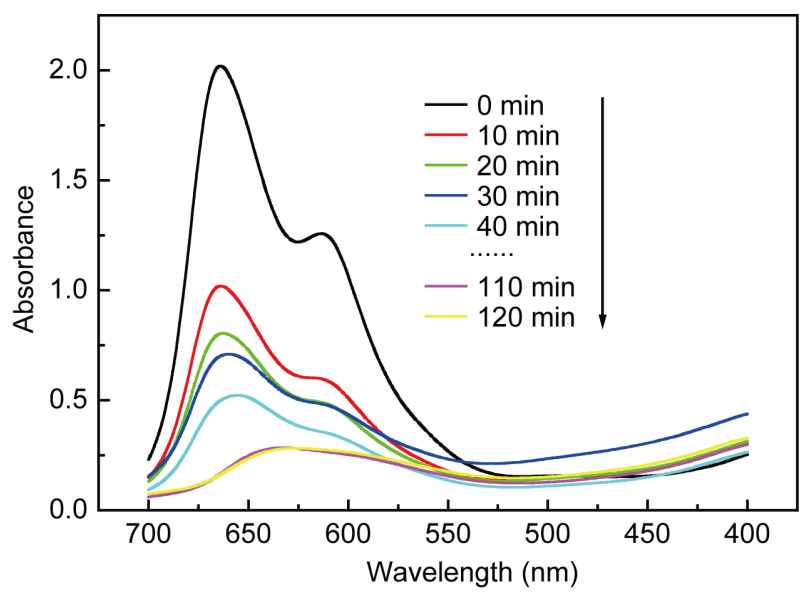

Figure 4. Visible absorption spectra of photo-degradation of methylene blue.

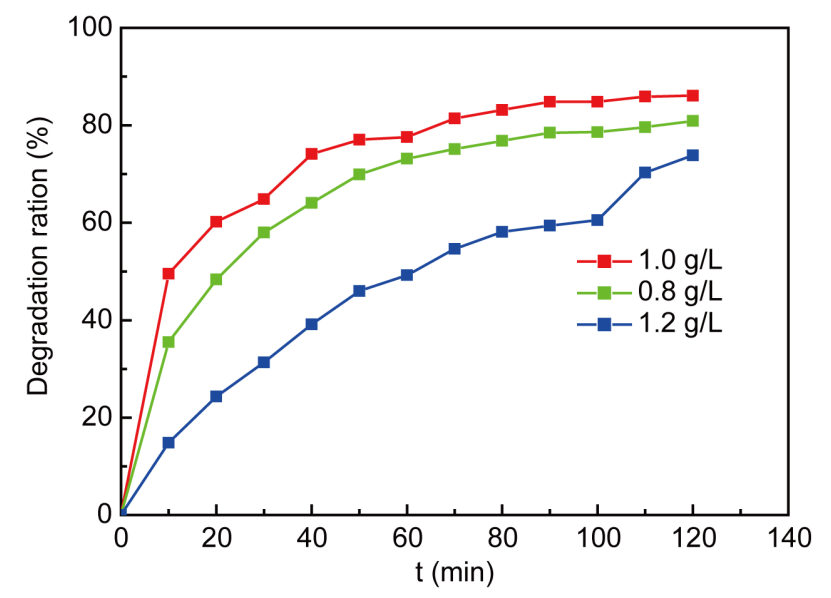

Figure 5. Effect of dosage of the $\mathrm{CdIn}_{2} \mathrm{~S}_{4}$ nanophotocatalyst on photocatalytic activity. 
For the application of visible light catalytic degradation of organic matter, efficient visible light catalyst should not only have narrow band gap energy, should also have the right band position. The valence oxidation potential should be high enough to be able to achieve the degradation of organic matter in hole, and position of the conduction band must also be appropriate to facilitate electron reduction. The $\mathrm{CdIn}_{2} \mathrm{~S}_{4}$ of band gap is $2.1-2.6 \mathrm{eV}$ less than $\mathrm{TiO}_{2}(3.2 \mathrm{eV})$, it has a good absorption in the visible range, it is a narrow band gap photocatalyst capable of absorbing visible light. The valence band of $\mathrm{CdIn}_{2} \mathrm{~S}_{4}$ can provide by $5 \mathrm{~s}$ orbital of In, is also provided by the $3 \mathrm{p}^{6}$ orbit of S. When the energy is sufficient to stimulate the light, 5 s of $\operatorname{In}^{3+}$ of $\mathrm{CdIn}_{2} \mathrm{~S}_{4}$ is likely to jump to the $3 \mathrm{~d}$ of $\mathrm{In}^{3+}$ space on the track, the energy required for this 5 s electron transition is lower than that of the $3 p$ of $\mathrm{S}$ electron transition. This $\mathrm{CdIn}_{2} \mathrm{~S}_{4}$ in the ultraviolet region and visible region is formed at least two absorption bands. The absorption band is mainly formed in the visible region feature by the $5 \mathrm{~d}$ of $\operatorname{In}^{3+}$ orbital electron transition to the $3 \mathrm{~d}$ of $\mathrm{Cd}^{2+}$ empty orbit.

The amount of catalyst has different impact on the photocatalytic degradation of organic pollutants. When the amount of the catalyst solution is too little, the photon energy of the light source cannot be fully utilized, the reaction rate is slower, when the amount of the catalyst is excessive, the scattering catalyst light affects the transmittance of the solution, so that the reaction rate is reduced.

$\mathrm{H}_{2} \mathrm{O}_{2}$ plays a role in initiating the reaction of the photocatalytic degradation process. $\mathrm{H}_{2} \mathrm{O}_{2}$ molecule has two hydrogen atoms linked to the oxygen atom, it is positively charged more than $\mathrm{O}_{2}$. It is also easier to capture the photogenerated electrons by optical excitation, to avoid recombination of photo generated electrons and light generating holes. The addition of $\mathrm{H}_{2} \mathrm{O}_{2}$ promoted the efficiency of photocatalytic degradation of $\mathrm{CdIn}_{2} \mathrm{~S}_{4}$. This may be caused by the following effects. On the one hand, $\mathrm{H}_{2} \mathrm{O}_{2}$ is able to capture the photogenerated conduction band electron, effectively inhibiting the recombination of electrons and holes, increase the number of highly active holes on the catalyst surface, and increase the oxidation rate of holes. On the other hand, $\mathrm{H}_{2} \mathrm{O}_{2}$ can generate a large number of hydroxyl radicals, therefore improve the degradation rate. However, excess $\mathrm{H}_{2} \mathrm{O}_{2}$ can not only improve the photocatalytic efficiency, instead, it will reduce the catalytic effect. So the amount of $\mathrm{H}_{2} \mathrm{O}_{2}$ added must beappropriate.

\section{Conclusion}

$\mathrm{CdIn}_{2} \mathrm{~S}_{4}$ nanophotocatalyst have been prepared by the method of direct feeding microwave synthesis, this method is simple. The results showed that the as-prepared catalyst has strong photocatalytic degradation ability. When the catalyst was $1.0 \mathrm{~g} / \mathrm{L}, \mathrm{C}\left(\mathrm{H}_{2} \mathrm{O}_{2}\right)$ was $3 \mathrm{~mL} / \mathrm{L}$, the degradation rate of $\mathrm{CdIn}_{2} \mathrm{~S}_{4}$ for methylene blue of $20 \mathrm{mg} / \mathrm{L}$ reached $86.06 \%$ in $120 \mathrm{~min}$.

\section{References}

[1] Fujishima, A. and Honda K. (1972) Electrochemical Photolysis of Water at a Semiconductor Electrode. Nature, 238, 37-38. https://doi.org/10.1038/238037a0 
[2] Cai, W., Zhao, Y.S. and Hu, J. (2011) Solvothermal Synthesis and Characterization of Zinc Indium Sulfide Microspheres. Journal of Materials Science \& Technology, 27, 559-562. https://doi.org/10.1016/S1005-0302(11)60108-4

[3] Kokate, A.V., Asabe, M.R., Delekar, S.D., et al. (2006) Photoelectrochemical Properties of Electrochemically Deposited $\mathrm{CdIn}_{2} \mathrm{~S}_{4}$ Thin Films. Journal of Physics and Chemistry of Solids, 67, 2331-2336. https://doi.org/10.1016/j.jpcs.2006.05.022

[4] Kokate A.V., Asabe M.R., Delekar S.D., et al. (2007) Structural, Optical and Eletrical Studies on Pulse Electro Deposited $\mathrm{CdIn}_{2} \mathrm{~S}_{4}$ Thin Films. Physica B: Condensed Matter, 390, 84-90. https://doi.org/10.1016/j.physb.2006.07.069

[5] Sawant, R.R., Rajpure, K.Y. and Bhosale, C.H. (2007) Determination of $\mathrm{CdIn}_{2} \mathrm{~S}_{4}$ Semiconductor Parameters by (Photo) Electrochemical Technique. Physica B: Condensed Matter, 393, 249-254. https://doi.org/10.1016/j.physb.2007.01.009

[6] Li, Y., Dillert, R. and Bahnemann, D. (2008) Perparation of Porous CdIn $\mathrm{S}_{4}$ Photocatalyst Films by Hydrothermal Crystal Growth at Solid/Liquid/Gas Interfaces. Thin Solid films, 516, 4988-4992. https://doi.org/10.1016/j.tsf.2007.10.004

[7] Zou, Z., Ye, J. and Arakawa, H. (2003) Photocatalytic Water Splitting into $\mathrm{H}_{2}$ and / or $\mathrm{O}_{2}$ under UV and Visible Light Irradiation with a Semiconductor PhotocatalystInter. International Journal of Hydrogen Energy, 28, 663-669. https://doi.org/10.1016/S0360-3199(02)00159-3

[8] Legrini, O., Oliveros, E. and Braun, AM. (1993) Photochemical Processes for Watertreatment. Chemical Reviews, 93, 671-698. https://doi.org/10.1021/cr00018a003

[9] Tang, Y., Yang, S., Zhu, Q. and Liao, X. (2016) The Microwave Synthesis and Photocatalytic Activity of $\mathrm{W}^{6+}$-Doped $\mathrm{TiO}_{2}$ Nanocomposite. Modern Research in Catalysis, 5, 45-49. https://doi.org/10.4236/mrc.2016.52005

\section{Submit or recommend next manuscript to SCIRP and we will provide best service for you:}

Accepting pre-submission inquiries through Email, Facebook, LinkedIn, Twitter, etc. A wide selection of journals (inclusive of 9 subjects, more than 200 journals)

Providing 24-hour high-quality service

User-friendly online submission system

Fair and swift peer-review system

Efficient typesetting and proofreading procedure

Display of the result of downloads and visits, as well as the number of cited articles

Maximum dissemination of your research work

Submit your manuscript at: http://papersubmission.scirp.org/

Or contact mrc@scirp.org 